\title{
Risk of suicide remains high fifteen years after deliberate self-harm
}

Hawton K, Zahl D, Weatherall R. Suicide following deliberate self-harm: long-term follow-up of patients who presented to a general hospital. Br J Psychiatry 2003;182:537-542.

QUESTION: What is the subsequent risk of suicide among patients presenting to a general hospital with an episode of self-harm?

\section{Design}

Retrospective longitudinal study.

Setting

General hospital, Oxford, UK; 1978-1997.

\section{Participants}

The original sample comprised all 12666 people who presented to the hospital after an episode of deliberate self-harm from 1978-1997. However, assessment was only possible for 11583 people.

\section{Assessment}

The Office for National Statistics (England and Wales), Central Services Agency (Northern Ireland) and the General Register Office for Scotland traced individuals to the end of 2000. Copies of death certificates were obtained. Those with a coroner's verdict of "suicide", "undetermined cause" or "accidental poisoning" were considered to have died from "probable suicide".

\section{Main outcome measures}

Risk of death due to probable suicide was calculated by the Kaplan-Meier method, and analysed by time since index episode of deliberate self harm, age and gender.

\section{Main results}

Overall, 300 people had died from probable suicide at the time of follow up.

Within one year of index episode: The overall risk of probable suicide was $0.7 \%(95 \%$ CI $0.6 \%$ to $0.9 \%)$. In males, the risk was $1.1 \%(95 \%$ CI 0.8 to 1.5$)$. In females, the risk was $0.5 \%(95 \%$ CI 0.4 to 0.7$)$.

Within 15 years of index episode: The overall risk of probable suicide was 3.0\% (95\% CI 2.6 to 3.4). In males, the risk was $4.8 \%$ (95\% CI 4.1 to 5.6). In females, the risk was $1.8 \%$ (95\% CI 1.5 to 2.2$)$. The hazard ratio for males relative to females was 2.8 (95\% CI 2.2 to 3.6).

Risk by age at time of index episode: The risk of suicide increased with advancing age at the time of the index episode of self-harm (hazard ratio for suicide in people aged $>55$ years $v$ people aged $10-24$ years $3.91,95 \%$ CI 2.71 to 5.62$)$.

K Hawton, Centre

Suicide Research,

University Department

of Psychiatry

Warneford Hospital,

Oxford OX3 7JX, UK.

keith.hawton@

psych.ox.ac.uk

\section{Conclusions}

The population who present with an episode of deliberate self-harm are at greatly increased risk of subsequent suicide. Absolute risks are higher for men and for people aged over 55 .

\section{COMMENTARY}

The conclusion of Hawton $e t$ al, namely that deliberate self harm is a predictor of successful suicide and therefore more attention should be paid to reducing that risk, flies in the face of my own impressions. The facts are clearly laid out that there is a rising trend in successful suicides in the subgroup of self-harmers, but I am not persuaded that the figures are either correct, nor that the conclusions are valid.

First, I note that over the decades, little hard evidence has been gathered on the efficacy of medical interventions in depression. Bennewith ${ }^{1}$ et al recently underlined the failure of general practice intervention to reduce future episodes of deliberate self harm. Numerous papers ${ }^{2-3}$ point out the failure of drugs to assist effectively. Finally an Australian paper ${ }^{4}$ purporting to show the efficacy of medical intervention in preventing suicide has been shot down in flames. This state of affairs awakens caution in reviewing further papers to this point.

Second, I wished that the figures in tabular form had been stratified for diagnostic criteria. I believe it makes a huge difference to the outcome if the patient suffers from a bipolar disorder or a manipulative personality disorder. I suspect, as my experience bears out, that deliberate self harm in a manipulative behavioural situation is a tool to manipulate the carer to an action that would not normally be suggested (admission to hospital perhaps?) and that a successful suicide in such a case would more likely represent a tragic accident.

Finally, perhaps the outcome should be measured with respect to societal conditions. It makes eminent sense - if at the same time a cutting criticism of society - to end one's life is there is not enough coal in the grate to keep you warm in your 80 's.

I found this paper thought provoking, and I look forward to further research as outlined.

Brian F Walker, MB ChB MRCGP General Practitioner Hong Kong

1 Bennewith O, Stocks N, Gunnell D, et al. General practice based intervention to prevent repeat episodes of deliberate self harm: cluster randomised controlled trial BMJ 2002; 324:1254.

2 Shorter E, Tyrer P. Separation of anxiety and depressive disorders: blind alley in psychopharmacology and classification of disease $B M J$ 2003;327: 158-160.

3 Melander H, Ahlqvist-Rastad J, Meijer G, Beermann B, Evidence b(i)ased medicine - selective reporting from studies sponsored by pharmaceutical industry: review of studies in new drug applications $B M J$ 2003;326:1171-1173.

4 in nall WD, Mant A, Mitchell PB, et al. Association between antidepressant prescribing and suicide in Australia 19912000: trend analysis. BMJ 2003; 326: 1008. 